\title{
The treatment of 'difficult' patients in a secure unit of a specialized psychiatric hospital: the patient's perspective
}

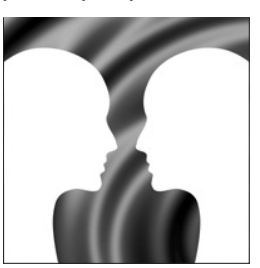

M. B OS ${ }^{1,5}$ R N M sc, N. KO OL-GOUDZWAAR D ${ }^{2,4}$ R N M s c, C. J. GAMEL RN PhD, B. KOEKKOEK ${ }^{3} \mathrm{RN} P \mathrm{Ph} \&$ B. VAN MEIJEL Rn PhDN ${ }^{1}$ Lecturer, Utrecht University of Applied Sciences, Department of Nursing, ${ }^{2}$ Associate Professor, Utrecht University, Utrecht, ${ }^{3}$ Researcher, ${ }^{4}$ Associate Professor, Research Group Mental Health Nursing, Department of Health, Welfare \& Sport, Inholland University of Applied Sciences, Amsterdam, ${ }^{5}$ Researcher, Palier, Parnassia Bavo Group, The Hague, ${ }^{6}$ Clinical Nurse Specialist, Pro Persona Mental Health Care, Wolfheze, ${ }^{7}$ Associate Professor, HAN University of Applied Sciences, Social Psychiatry \& Mental Health Nursing Research Group, Nijmegen, The Netherlands

Keywords: counter transference, 'difficult' patients, recovery, structure, treatment

Correspondence:

M. Bos

Bandoengstraat 76

3531RN Utrecht

The Netherlands

E-mail: mathildebos@planet.nl

Accepted for publication: 30 August 2011

doi: $10.1111 / \mathrm{j} .1365-2850.2011 .01827 . \mathrm{x}$

\section{Accessible summary}

- Some patients in psychiatric hospitals are considered difficult to treat. Their ongoing conflicts with nurses and other health care professionals can result in a deadlock in their treatment.

- In these cases, patients can be transferred to another specialist hospital. The research described below was conducted in such a specialist psychiatric hospital. The researchers asked the patients how they felt about their treatment and how it helped them recover.

- The patients found the treatment very strict: they had to make commitments and to follow numerous rules concerning eating times, privileges and safety. Also the professionals pointed out the patient's own responsibility for the success of the treatment.

- The patients reported that the treatment had helped them considerably. Their anxiety, aggression and other symptoms had decreased. Their confidence increased, and they perceived their future with greater optimism.

\begin{abstract}
The aim of this study is to obtain insight, from a patient's perspective, into the results and essential components of treatment in specialist settings for so-called 'difficult' patients in mental health care. In cases where usual hospital treatment is not successful, a temporary transfer to another, specialist hospital may provide a solution. We investigated which aspects of specialist treatment available to 'difficult' patients are perceived as essential by the patients and what are the results of this treatment in their perception. A qualitative research design based on the Grounded Theory method was used. To generate data, 14 semi-structured interviews were held with 12 patients who were admitted to a specialist hospital in the Netherlands. Almost all respondents rated the results of the specialist treatment as positive. The therapeutic climate was perceived as extremely strict, with a strong focus on structure, cooperation and safety. This approach had a stabilizing effect on the patients, even at times when they were not motivated. Most patients developed a motivation for change, marked by a growing and more explicit determination of their future goals. We concluded that a highly structured treatment environment aimed at patient stabilization is helpful to most 'difficult' patients.
\end{abstract}




\section{Introduction}

Some psychiatric patients are perceived as extremely difficult by their treatment team because they are demanding or exhibit impulsive, agitated, suicidal, aggressive or selfdestructive behaviour. Such conduct can evoke strong emotions of counter transference in the care providers, including feelings of irritation, frustration and powerlessness (Cleary et al. 2002, James \& Cowman 2007). Caregivers devote considerable time and energy upon a small group of patients that is perceived as 'difficult' (Koekkoek 2011). The 'difficult' label is associated with professional pessimism, passive treatment and possible discharge or referral from care, as well as with multiple diagnoses and diagnostic uncertainty (Koekkoek 2011, Koekkoek et al. 2011). The treatment team is often divided regarding how to approach and treat these patients (Sonneborn \& Dijkstra 2003, Koekkoek et al. 2006). Adler (2006) and Koekkoek et al. (2006) conclude that the 'difficult' patient may be hard to define, but the concept is well known in many clinical settings. This is the reason that we use the label 'difficult' in this paper - even though we realize this term may be subject to discussion.

It is clear from research that problems with the treatment and care of this group of patients are traceable not only to patient-specific factors, but also to factors more closely connected to the care providers or the team and the organization as a whole (Markham \& Trower 2003, Koekkoek et al. 2006). Various studies (Breeze \& Repper 1998, Koekkoek et al. 2006, Stringer et al. 2008) show that patients perceived as difficult can easily become involved power struggles with their care providers, revolving, for example, around the patient's disruptive behaviour, the administration of medication, the granting of privileges or (forced) admission. This struggle may even escalate to such an extent to disrupt the entire therapeutic relationship, causing the treatment to come to a standstill (Breeze \& Repper 1998, Koekkoek et al. 2006, Mayer 2008). In such situations, the need to prevent and overcome the deadlock is one of the main objectives of treatment.

Substantial research has been conducted into the common characteristics and problems of 'difficult' patients, but pertinent cofactors in the domain of the care providers and in the domains of treatment or the treatment team are underrepresented in research to date. It is generally the patients who are held accountable for evoking strong counter transference reactions as well as for the deadlock in their treatment process (Koekkoek et al. 2006). From the limited research conducted of these patients' perspectives it is known that they lack recognition from caregivers (Breeze \& Repper 1998, Koekkoek et al. 2010).
The Netherlands has a number of specialist psychiatric hospitals where patients are referred following seriously disrupted therapeutic relationships. The present research was conducted in one of these specialist hospitals, which runs a specialized, intensive and multidisciplinary treatment programme. This programme specifically focuses on establishing a therapeutic relationship and on regulating disruptive behaviours.

The therapeutic approach in this specialist hospital is more structured than in general psychiatric hospitals and there is a strong focus on therapeutic alliance and safety. This approach is partly based on the theory of Rogers, as described by van Kessel \& van der Linden (1991), which focuses on communication and interaction. The importance given to the strict structure is based on experience with the treatment of patients referred to this hospital.

From evaluation research, it is evident that such specialist treatment often overcomes the deadlock in treatment (Sonneborn \& Dijkstra 2003, Kool et al. 2009). Little is known about the experiences of patients during their treatment in a specialist hospital.

The aim of the present study was to examine how patients experience the specialist treatment and which elements of the treatment they perceive to be essential. Thus, a theoretical model was developed concerning the perceived treatment results.

\section{Research method}

\section{Design}

The objective was to use the patient's perspective as a basis for developing a theoretical explanation of the perceived result. Grounded Theory (GT) is a qualitative method that aims to develop theory grounded in empirical data (Glaser \& Strauss 1967). Holloway \& Wheeler (2006) describe that GT is derived from the insights of symbolic interactionism which explains how individuals take account of each other's acts, interpret them and organize their own behaviour. GT is widely used in areas in which current (theoretical) knowledge is limited and is considered particularly useful in the study of human behaviour and social roles. GT is a suitable design for this research study because theoretical knowledge is minimal concerning the central topic, namely the perspectives, feelings and experiences of 'difficult' patients in a specialist treatment setting.

\section{Setting}

The research was conducted at a secure 16-bed unit within a specialist psychiatric hospital. Most patients were admitted involuntarily and had a long history of admissions and 
treatment. The treatment team at the specialist hospital consisted of nurses, occupational therapists, social workers, a physician, psychologists and several psychiatric therapists. Each patient had his or her own therapist and primary nurse. The emphasis of treatment was upon structure, cooperation and safety.

The specialist hospital's medical ethical committee approved the study.

\section{Respondents}

The sample comprised all patients admitted, during the period of research (convenience sample), who were able and willing to participate and who had reached the final phase of their treatment. Patients were informed of the study both in writing and verbally. Patients were not rewarded for their participation in this study and refusal did not have consequences for further treatment. Anonymity of the patients and confidentiality of the research data were guaranteed. Written informed consents were requested and obtained.

\section{Data collection}

One of the researchers (M. B.) collected all data. This researcher had no connection whatsoever to the hospital. Semi-structured interviews were used, recorded on audiotape and transcribed verbatim. Field notes were made after every interview and discussed with the second researcher (N. K., peer debriefing, reflexivity). Memos were written throughout the coding process to record emerging conceptual links and other observations about the data. Each interview lasted approximately $1 \mathrm{~h}$. The topic list was constructed on the basis of the existing literature about 'difficult' patients and disrupted therapeutic relationships. Also the hospital's treatment approach provided a source for interview topics. The opening question was: 'Can you tell me how you were doing when you first came here?' The next question was: 'Can you tell me how you are doing now?' This provided pertinent information as to how patients perceived the results of their treatment. Then, the contributing factors to these treatment results were explored, as well as their personal experiences concerning their treatment. Interview topics were discussed randomly, depending on the patients' responses. Respondents were asked about certain topics if they had not mentioned them at all. The topic list was expanded whenever respondents mentioned new items that were important to them and in response to new insights concerning plausible topics originating from the analysis of previous interviews.
Two respondents were invited to a second interview in order to explore the developed theory (theoretical sampling, member check) and to answer more in-depth questions.

To obtain more insight into the respondents' characteristics, diagnoses and Global Assessment of Functioning scores were collected (American Psychiatric Association 2000).

Evaluation forms which respondents filled in at discharge were examined (methodological triangulation).

\section{Analysis}

The constant comparative method was used to analyse data. Data collection and data analysis took place concurrently in an iterative process, typical to the GT method (Holloway \& Wheeler 2006). The derived items were grouped into several categories, for example, 'structure', 'power struggle' and 'future'. The links between the categories formed the basis for theory development. These processes were discussed twice in the research group (M. B., N. K., B. V. M.) during this investigation. Contradictions that were identified during the analysis and new questions that emerged were presented in subsequent interviews (member check). After 10 interviews, all data could be allocated to the existing categories and no new data emerged.

\section{Results}

During the research period, 20 patients were eligible for the study because they had reached the final phase of their treatment. A total of 14 interviews were held with 12 respondents: eight women and four men between the ages of 17 and 45. Eight patients were unable or unwilling to participate.

All respondents, except one, were admitted involuntarily. The period of their stay varied from 7 weeks to 13 months. A total of 32 Diagnostic and Statistical Manual of Mental Disorders IV diagnoses ${ }^{1}$ were made for the 12 respondents interviewed: 22 on Axis I and 10 on Axis II. Nine of the 12 respondents were diagnosed with multiple disorders. Diagnoses changed and new diagnosis were added during treatment in the specialist hospital.

\footnotetext{
${ }^{1}$ Axis I: schizophrenia $(n=3)$, schizo affective disorder $(n=2)$, depressive disorder $(n=2)$, substance-related disorder $(n=4)$, bipolar disorder $(n=2)$, dissociative disorder $(n=2)$, eating disorder $(n=2)$, posttraumatic disorder $(n=2)$, generalized anxiety disorder $(n=1)$, reactive attachment disorder $(n=1)$, gender identity disorder $(n=1)$. Axis II: personality disorder, not otherwise specified $(n=2)$, borderline personality disorder $(n=6)$, antisocial personality disorder $(n=1)$, narcissistic personality disorder $(n=1)$.
} 
Figure 1

Relationships among elements identified as essential by patients for stabilization and positive change

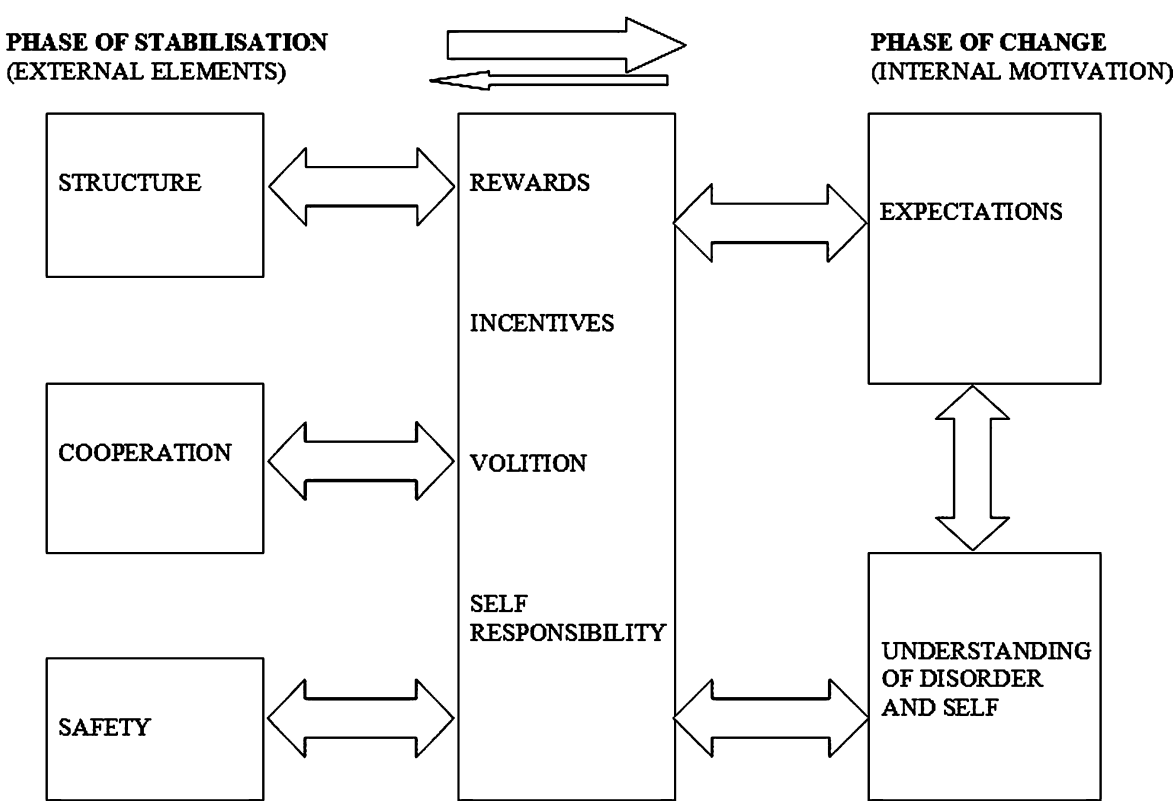

On admission, the respondents' average Global Assessment of Functioning score (American Psychiatric Association 2000) was 30, indicating a very low level of general functioning.

With the evaluation form, completed immediately before discharge, respondents made a judgment of the treatment that they had received as a whole. The mark given indicates their overall satisfaction with the specialist treatment (scale 1-10; 1 was the lowest score and 10 the highest). One of the respondents gave ' 1 ', a figure that we have regarded as an outlier. The average mark of the remaining respondents was 7.3.

Firstly a description of how the respondents experienced the results of their treatment is presented. Then the stabilization phase and the change phase are described. These phases emerged from the analysed data and thus were used to group the results. Those treatment elements considered essential by patients are addressed within each treatment phase. The relationships between those elements identified as being essential by patients for stabilization and positive change are described in Fig. 1 which presents the theory developed in this study.

\section{Perceived treatment result}

All but two of the respondents reported that they felt better at the end of the specialist treatment. Their destructive behaviour had abated and they felt less anxious, suicidal and/or sedated. Looking back to the time of admission, all but one of the respondents rated their psychological condition as poor, with little or no control over their own behaviour and emotions. At the end of the treatment, 10 patients experienced stabilization as a main result and eight of them experienced change as well. Most respondents credited themselves for the positive result of the treatment and they were proud of their achievements. These respondents mentioned that they were not treated at all because according to them - hardly any therapeutic sessions had taken place. The daily 'therapeutic climate' was not considered by them as 'treatment'.

\section{Phase of stabilization}

Most of the respondents reported that they had no motivation for treatment on admission to the specialist hospital. Structure, cooperation and safety were mentioned in virtually all interviews as key features of their new environment. The respondents perceived these aspects as having a stabilizing effect.

\section{Structure}

In the other hospital I could do whatever I wanted ... I always could get away with that. This was not good for me, oh no $(\ldots)$ In this hospital you really have to follow all the tiny rules. (Respondent 6)

A strict structure was mentioned by all as the unit's central feature. All respondents were in a position to compare the structure with that of other hospitals or institutions where they had been before and all qualified their unit in the specialist hospital as having the strictest structure.

The respondents reported that the consequences of their behaviour were clear, as these were outlined in their treat- 
ment plan. It was their experience that the treatment plan, as well as discipline in general, were strictly enforced; the rules were firm, and no crossing of boundaries was permitted.

Compliance with the requirements of the treatment plan was rewarded with privileges and this was regarded as an incentive by patients. By contrast, a patient's lack of cooperation led to the withdrawal of privileges, which several respondents regarded as punishment. The treatment team consistently emphasized patients' volition and patients were therefore responsible for their own choices and actions. The strict structure also evoked criticism: eight of the 12 respondents resisted the rules in place and became involved in a struggle for power with the treatment team.

\section{Cooperation}

I could start again and again, my behaviour was forgiven $(\ldots$ ). They limited my behaviour but I didn't feel rejected as a human being. (Respondent 8 )

I must take the initiative in everything, it's not coming from them. That's not what I call cooperation, when I'm solely responsible. I'm anyway totally fed up with the quote 'it's your own responsibility'. (Respondent 5)

A willingness to cooperate with the team was stated by respondents as an essential condition for progress. They also reported that a strict structure and a strict allocation of roles were maintained in the context of that cooperation. The matters discussed with their primary nurse, for example, were different from those discussed with other nurses or with the treating therapist. A 30-min meeting with the primary nurse was scheduled each week in order for the patients to discuss treatment issues. Primary nurses were responsible for coordinating the individual programmes and act as intermediaries between patients and staff members. The role of the primary nurse was considered to be very important: seven of the 12 respondents explicitly stated that a relationship of trust had developed during their stay at the hospital. In each shift, there was one designated nurse with whom patients could, on request, have a consultation of up to $15 \mathrm{~min}$. It was mostly left to the patients to take the initiative. The only issues permitted to be raised in such consultations were related to the management of problems in everyday life. The patients were asked to contribute their own solutions to address, for example, anxiety or threatening (auto-) aggression. This focus on the patients' own responsibility encouraged initiative, but also evoked resistance. Some patients explicitly reported that the need to take action of their own was not how they perceived cooperation. They had expected the nurses to provide more input in helping them resolve the problems in question.
According to the respondents, the team closely monitored whether a balance existed between distance and nearness in the patients' relationship with both the care providers and the fellow patients. Of the 12 respondents, nine criticized this situation and qualified the team as detached. Other adjectives used were authoritative, antiaccommodating and indifferent. In some respondents, this evoked feelings of loneliness. However, none of the respondents made any mention of feeling rejected by the team. Being rejected was frequently mentioned by the respondents when talking about their experiences in other hospitals. Despite the detachment felt by the nine respondents, they stated explicitly that some of the team members did show interest and understanding. Some of them specifically described the conduct they valued, using qualifications such as: 'they create a good atmosphere', 'they talk in a soft voice', 'they look you in the face', 'they want to help' or 'they do not just say that it is your own responsibility'. The respondents repeatedly remarked how much they appreciated the company of nursing staff in the common area and how much they liked watching TV or drinking coffee together with the nurses. The simple act of being there was sufficient for the patients to feel that the staff showed an interest in them as a person.

\section{Safety}

And they always ask what is needed to keep things safe. (Respondent 3)

For most respondents, one of the main symptoms for being admitted to the specialist hospital was their destructive behaviour, which jeopardized not only the patients' own safety, but sometimes also that of their social environment. All respondents reported that safety was a matter of utmost priority, and that immediate action was taken whenever their safety or that of others was under threat. In this context, too, the patients' own responsibility was highlighted. Learning to stay safe was a major goal of treatment. Accordingly, the patients had an important say in the application of coercive measures, such as taking time-outs or putting away dangerous objects.

Respondents generally claimed that they felt safe at the unit, even though virtually all of them reported incidents of aggression in which they were personally involved (either actively or passively).

In short, the phase of patient stabilization was supported by rewarding the patient with incentives such as receiving privileges for cooperation with the treatment plan. The positive and negative consequences of disruptive behaviour formed stimuli for the patients to adjust their behaviour (Fig. 1). 


\section{Phase of change}

You must want to change; if you do not want that, it will all be for nothing. (Respondent 1 )

During the phase of change, the motivation of the patients was more intrinsic, resulting in fewer struggles for power and more room for change. Eight respondents described how they had decided in the course of treatment to support the treatment plan and that their cooperation with the team had consequently become voluntary in nature, allowing room for a more constructive form of cooperation. Several respondents stressed the importance of being intrinsically motivated. The eight respondents referred to above mentioned a gradual increase in self-esteem and understanding of their disorder. They were able to set treatment goals of their own and define their expectations for the future.

\section{Self-esteem and understanding of the disorder}

I am prouder of myself $(\ldots$ ) because I believe that I do matter, that I will not be judged, I stick by the rules, I am becoming me again, I am more accepting of myself. (Respondent 12)

Whenever a patient succeeded in creating more constructive relationships and complying with the treatment plan, their self-confidence grew. Respondents perceived this development as a personal accomplishment. Their new sense of self-esteem and understanding of the disorder helped the process of recovery. One of the reasons for this change was the constant reminder by team members of what they were doing and what the consequences were (positive and negative). This direct feedback gave the patients direction and provided the patients with better insight and gave them more confidence.

\section{Expectations}

So this is what drives me: I want to go home and live my own life. (Respondent 8)

In the phase of change, the respondents also mentioned their expectations of the future as a major motivation for resuming responsibility. Respondents jumped up and spoke ardently of their future plans and ideas whenever the topic was brought up in the interviews. The expectations fed the patients' intrinsic drive towards change. For two of the respondents, who had been separated from the other patients for a long time, the time-out period broke the pattern of struggle and resistance. Both had reflected on the question of what they actually wanted with their lives during that period and had made a 'deeply felt' choice (intrinsic motivation) to work on change and move towards recovery.
The respondents generally expressed feelings of ambivalence during the phase of change. Even after the decision to change was made, intrinsic motivation was not present constantly and there were many relapses to old habits of thought and behaviour. Nine of the 12 respondents indicated that it had been very difficult for them to appeal to their own responsibility and their own intrinsic motivation. Those difficulties evoked a sense of powerlessness in them.

It is easy for them to say 'It's your own responsibility', but it is not as simple as that (...) If it were that easy to turn things around, I would have done so ages ago. (Respondent 6)

\section{Discussion}

Patients experienced the supportive and strictly structured treatment setting as a positive environment for stabilization and change. They saw the hospital's approach regarding structure, cooperation and safety reflected in their daily life in the hospital. The treatment plan discussed with patients was commensurate with this approach and commitments were more strictly enforced than had been the case in previous, failed treatment settings. Koekkoek et al. (2006) and van Meekeren (2009) contend that the existence of a comprehensible, coherent and unambiguous treatment plan that is strictly enforced, also in the context of the daily interactions between staff and patient, is a prerequisite for the treatment of this group of patients. Sonneborn \& Dijkstra (2003) maintain that the emphasis in the treatment of difficult patients should be on safety and on taking responsibility for one's own actions. These aspects are clearly present in the treatment setting studied in this research. The positive treatment result experienced by the interviewed patients might be explained by the need they have for a clear external structure as long as they have little 'internal structure', that is, control over their own emotions, behaviour and motivation.

Surprisingly, most respondents reported that they did not experience the strict regime in the hospital as constituting therapeutic treatment. They associated treatment with therapists and therapeutic sessions about problems and traumas. The therapeutic climate, in which daily life and interactions are the main ways through which new behaviours and coping strategies are practiced, was not recognized as treatment. This, together with repeatedly being reminded of their own responsibility, resulted in patients' perception that recovering occurred because of their own efforts. This was very beneficial for their self-esteem.

Although some respondents reported feeling lonely, none of them felt in any way personally rejected by the treatment team despite their qualification of the care pro- 
viders as being detached and authoritative. This is a remarkable fact, because almost all of the respondents stated that they felt rejected during former treatments in other hospitals. This rejection was one of the main reasons respondents gave in explaining the necessity for referral to this specialist hospital. This corresponds with some of the aspects associated with the label 'difficult': professional pessimism and referral out of care (Koekkoek 2011, Koekkoek et al. 2011). Koekkoek et al. (2010) describe optimal clinical practice as the space between doing too much (toxic high) and too little (toxic low). This could be the space patients described in this study: detached but not rejecting. The question remains whether the care providers deliberately adopted this attitude to support the treatment process or whether they used it as a coping strategy, to manage their feelings of counter transference inevitably surfacing in working with this group of patients, which can result in rejecting the patient.

According to Koekkoek et al. (2006), little is known about coping strategies of health care professionals. Bowers et al. (2005) found that staff working with dangerous patients with a personality disorder, needed a clear philosophy and treatment regime, staff training programmes and supervision, to maintain a positive attitude towards patients. The respondents reported that a minority of the care providers did succeed in combining strict enforcement of the rules with an attitude of understanding and empathy. In their view, such a combination was a major condition for engaging in a constructive relationship with the care providers.

Respondents not only expressed their satisfaction with their treatment, but also levelled criticism. Nonetheless, virtually all respondents rated their treatment as good. This confirmed the strong ambivalence that respondents experienced, which emerged in many of the interviews. Koekkoek et al. (2006) identify ambivalence as a feature that is typical of a specific group of 'difficult' patients who benefit least from regular treatment programmes. A tentative conclusion that can be drawn in this regard is that these patients (initially) fight the therapeutic climate which they need at the same time to recover.

The expectations that the respondents harboured for the future emerged unexpectedly as a strong motivation for them to take back responsibility for their own actions. There is no reference to this aspect in the literature to date. These expectations may be more important to patients than care providers realize.

It is inferred from these results that the care providers at the referring hospitals must take a new attitude towards these patients after the patients' return to the referring hospital. In order to prevent relapses, the team must realize that they are also responsible for the deadlock and that they and the patient together share responsibility for preventing, and breaking new deadlocks. Support and advice from the specialist hospital is crucial to the continuation of positive treatment results. Follow-up research on this transition care is needed.

\section{Strength and limitations}

More than half of the available research population was interviewed. There were many similarities in the respondents' descriptions of the therapeutic climate. This made it possible to draw preliminary conclusions on the patient's perspective of the treatment programme offered at the specialist hospital. However, only 12 patients, within one special treatment setting were involved in our study, so that any extrapolation of the outcomes to other hospitals must be approached with caution.

\section{Conclusion and recommendations}

The aim of this study was to describe how patients experienced the specialist treatment and which elements of the treatment were perceived as essential for obtaining positive results. The conclusion is that 'difficult' patients exhibiting highly disruptive behaviour can be treated if the treatment team responds adequately to the needs of these patients in a supportive and structured environment. Key to the treatment of 'difficult' patients is the drafting of a clear treatment plan and strictly enforcing compliance with that plan. Establishing a therapeutic relationship in which ensuring that the patient does not feel rejected was experienced as important, as was the allocation of a primary nurse to each patient.

\section{Further research}

Further research is needed to:

- assess the extent to which patients are able to sustain the improvements that have been made after discharge from the secure unit of a specialized hospital;

- obtain greater insight into 'difficult' patients' perspectives about the care provided and the treatment outcomes achieved in different health care settings;

- explore the day-to-day interactions between nurses and patients;

- obtain insight in the therapeutic influence of patients' expectations of the future;

- acquire greater understanding of the difference in staff members coping strategies in managing the dynamics of transference and counter transference in the therapeutic relationship with 'difficult' patients. 


\section{Implications for practice}

These results may have a major impact upon daily practice, especially in situations when 'difficult' patients challenge the efforts of caregivers in attempting to nurture a therapeutic partnership with them. The findings contain descriptions of intervention strategies that staff members can use to manage transference and counter transference as well as to establish positive change with so called 'difficult' patients.

\section{Acknowledgments}

The authors wish to express their gratitude to the patients and staff of Palier, Intensive Treatment Centre in The Hague for their valuable contribution to this research project.

\section{References}

Adler D.A. (2006) Difficult patients: within themselves and with caregivers. Psychiatric Services $57,767$.

American Psychiatric Association (2000) Diagnostic and Statistical Manual of Mental Disorders, 4th edn. DSM IV, American Psychiatric Association, Washington, DC.

Bowers L., Carr-Walker P., Paton J., et al. (2005) Changes in attitudes to personality disorder on a DSPD unit. Criminal Behaviour and Mental Health 15, 171-183.

Breeze J.A. \& Repper J. (1998) Struggling for control: the care experiences of 'difficult' patients in mental health services. Journal of Advanced Nursing 28, 1301-1311.

Cleary M., Siegfried N. \& Walter G. (2002) Experience, knowledge and attitudes of mental health staff regarding clients with a borderline personality disorder. International Journal of Mental Health Nursing 11, 186-191.

Glaser B. \& Strauss A. (1967) The Discovery of Grounded Theory. Aldine, Chicago, IL.

Holloway I. \& Wheeler S. (2006) Qualitative Research in Nursing. Blackwell Science, Oxford.
James P.D. \& Cowman S. (2007) Psychiatric nurses' knowledge, experience and attitudes towards clients with borderline personality disorder. Journal of Psychiatric and Mental Health Nursing 14, 670-678.

van Kessel W. \& van der Linden P. (1991) Leerboek Gesprekstechniek: De Cliëntgerichte Benadering. [Communication: The PatientCentred Approach.]. (Swildens H., red). Acco, Amersfoort/Leuven.

Koekkoek B. (2011) Ambivalent Connections: Improving Community Health Care for NonPsychotic Chronic Patients Perceived As 'Difficult'. Radboud Universiteit, Nijmegen.

Koekkoek B., van Meijel B. \& Hutschemaekers G. (2006) 'Difficult patients' in mental health care: a review. Psychiatric Services 57, 795-802.

Koekkoek B., van Meijel B., van Ommen J., et al. (2010) Ambivalent connections: a qualitative study of the care experiences of non-psychotic chronic patients who are perceived as 'difficult' by professionals. BMC Psychiatry 10, 96107.

Koekkoek B., Hutschemaekers G., van Meijel B., et al. (2011) How do patients come to be seen as 'difficult'? A mixed-methods study in community mental health care. Social Science \& Medicine 72, 504-512.
Kool N., van Meijel B., Koekkoek B., et al. (2009) The Centre for Intensive Treatment of Disruptive Relationships in Psychiatry, Preliminary Evaluation. Parnassia Bavo Group, The Hague. Markham D. \& Trower P. (2003) The effects of the psychiatric label 'borderline personality disorder' on nursing staff's perception and causal attributions for challenging behaviours. The British Journal of Clinical Psychology 42, 243256.

Mayer M.L. (2008) On being a 'difficult' patient. Health Affairs (Project HOPE) 27, 1416-1421. van Meekeren E. (2009) De multidisciplinaire richtlijn persoonlijkheidsstoornissen; een kritische beschouwing. [The multidisciplinary guideline personality disorders: a critical review.]. Maandblad Geestelijke Volksgezondheid 64, 286-297.

Sonneborn C.K.M. \& Dijkstra J.B. (2003) Moeilijke psychiatrische patiënten: een retrospectief follow-uponderzoek. [Difficult patients in psychiatry: a retrospective follow-up study.]. Tijdschrift Voor Psychiatrie 45, 5-14.

Stringer B., van Meijel B., de Vree W., et al. (2008) User involvement in mental health care: the role of nurses. A literature review. Journal of Psychiatric and Mental Health Nursing 15, 678683. 